\title{
An Extended Finite Element Model for Fluid Flow in Fractured Porous Media
}

\author{
Fei Liu, ${ }^{1}$ Li-qiang Zhao, ${ }^{1}$ Ping-li Liu, ${ }^{1}$ Zhi-feng Luo, ${ }^{1}$ Nian-yin Li, ${ }^{1}$ and Pei-shan Wang ${ }^{2}$ \\ ${ }^{1}$ State Key Laboratory of Oil \& Gas Reservoir Geology and Exploitation, Southwest Petroleum University, \\ Chengdu, Sichuan 610500, China \\ ${ }^{2}$ Exploration Utility Department, Southwest Oil and Gas Field Company, PetroChina, Chengdu, Sichuan 610000, China
}

Correspondence should be addressed to Fei Liu; liufei10628@aliyun.com

Received 31 December 2014; Accepted 5 January 2015

Academic Editor: B. Rush Kumar

Copyright (C) 2015 Fei Liu et al. This is an open access article distributed under the Creative Commons Attribution License, which permits unrestricted use, distribution, and reproduction in any medium, provided the original work is properly cited.

\begin{abstract}
This paper proposes a numerical model for the fluid flow in fractured porous media with the extended finite element method. The governing equations account for the fluid flow in the porous medium and the discrete natural fractures, as well as the fluid exchange between the fracture and the porous medium surrounding the fracture. The pore fluid pressure is continuous, while its derivatives are discontinuous on both sides of these high conductivity fractures. The pressure field is enriched by the absolute signed distance and appropriate asymptotic functions to capture the discontinuities in derivatives. The most important advantage of this method is that the domain can be partitioned as nonmatching grid without considering the presence of fractures. Arbitrarily multiple, kinking, branching, and intersecting fractures can be treated with the new approach. In particular, for propagating fractures, such as hydraulic fracturing or network volume fracturing in fissured reservoirs, this method can process the complex fluid leak-off behavior without remeshing. Numerical examples are presented to demonstrate the capability of the proposed method in saturated fractured porous media.
\end{abstract}

\section{Introduction}

Flow models estimating the flow in fractured porous media mainly include the equivalent continuum model [1], dual continuum model [2], discrete fracture model [3], and discrete fracture network model $[4,5]$. These models fall into two categories: continuum models and discrete fracture models. In the equivalent continuum model, a representative elementary volume (REV) is required, the fractures are assumed to distribute regularly, and the equivalent permeability is hard to determine [6], which make it unavailable while several large fractures existed such as hydraulic fractures, complex fractures network, and big faults. Dual continuum model cannot describe the flow in fracture because the fractures are not treated explicitly in it $[7,8]$. Discrete fracture network model is more real and gains worldwide concern recently because the fractures are treated explicitly and the effect of one crack on the whole flow could be considered [9]. Yao Jun et al. [10] presented a two-dimensional two-phase finite element flow model based on explicit treating of discrete fracture network. In the model, matrix domain is partitioned using triangle finite element method (FEM) grids while large fracture is partitioned using one-dimensional line elements. The grids are required to match the explicit fractures and nodes are needed on both fracture intersections and crack tips to better solve such static fracture problems. Moreover, tedious remeshing is necessary for propagating fractures [11]. The extended finite element method (XFEM) is an effective approach to avoid remeshing and is now widely used to solve discontinuity problem [12-14]. Khoei et al. [15] simulated the coupled thermohydromechanical (THM) model for impermeable discontinuities in saturated porous medium with XFEM. Mohammadnejad and Khoei [16] presented a coupled hydromechanical (HM) model with combined XFEM and cohesive crack. Lamb [17] and Lamb et al. [18] combined XFEM with the dual-porosity and dual-permeability model to describe the fluid flow, deformation, and fracture propagation in fractured porous medium. By virtue of XFEM and lower-dimensional interface elements, Watanabe et al. [19] researched coupled HM problems in discrete fractures 
porous media systems. Based on the methodology of XFEM, in this paper, the pressure field is enriched by the absolute signed distance and appropriate asymptotic functions to develop an XFEM model for fluid flow in fractured porous medium. The results demonstrate that the XFEM is an efficient method for simulating fluid flow in fractured porous medium with nonmatching grids, especially when the fissure is propagating, such as hydraulically driven fractures.

\section{Governing Equation}

The strong form as well as the associated weak form of governing equations for fluid flow inside both matrix domain and fracture domain is demonstrated in the section.

2.1. Strong Form. According to the law of mass conservation, the continuity equation for flow in the matrix domain is written as

$$
\left(\frac{\alpha_{m}-\phi_{m}}{K_{s}}+\frac{\phi_{m}}{K_{w}}\right) \frac{\partial p_{m}^{w}}{\partial t}+\nabla \cdot\left(-\frac{\mathbf{K}_{m}}{\mu_{w}} \nabla p_{m}^{w}\right)+Q_{w s t c} B_{w}=0
$$

Similarly, the continuity equation for flow inside the fracture domain is given by

$$
\frac{1}{K_{w}} \frac{\partial p_{f}^{w}}{\partial t}+\nabla \cdot\left(-\frac{K_{f}}{\mu_{w}} \nabla p_{f}^{w}\right)=0
$$

where $\alpha_{m}$ is the Biot constant, $\varphi_{m}$ is the porosity of matrix rock, $K_{s}$ is the bulk modulus of solid phase, $\mathrm{Pa}, K_{w}$ is the bulk modulus of pore fluid, $\mathrm{Pa}, p_{m}^{w}$ is the pore fluid pressure in matrix, $\mathrm{Pa}, t$ is time, $s, \mathbf{K}_{m}$ is the permeability tensor of matrix, $\mathrm{m}^{2}, \mu_{w}$ is the pore fluid viscosity, Pa.s, $Q_{w s t c}$ is the source/sink term on the ground, $\mathrm{m}^{3} /\left(\mathrm{m}^{3} \cdot \mathrm{s}\right), B_{w}$ is the bulk coefficient, $p_{f}^{w}$ is the fluid pressure in fracture, $\mathrm{Pa}$, and $K_{f}$ is the permeability of fracture, $\mathrm{m}^{2}$, calculated according to the following formula [20]:

$$
K_{f}=\frac{1}{f} \frac{b^{2}}{12}
$$

where $f$ is a morphological parameter accounting for the difference between real fracture and ideal parallel fracture, ranging from 1.04 to $1.65 . b$ is the mechanical width of fracture, $m$.

2.2. Weak Form. In order to deduce the weak form of governing equations, a two-dimensional domain $\Omega$ bounded by the boundary $\Gamma$ is considered as shown in Figure 1. Natural fracture with high permeability inside $\Omega$ is regarded as a onedimensional discontinuous line, because the fracture width is much less than the length. $\Gamma_{f}^{+}$and $\Gamma_{f}^{-}$are used to represent two faces of the fracture, respectively.

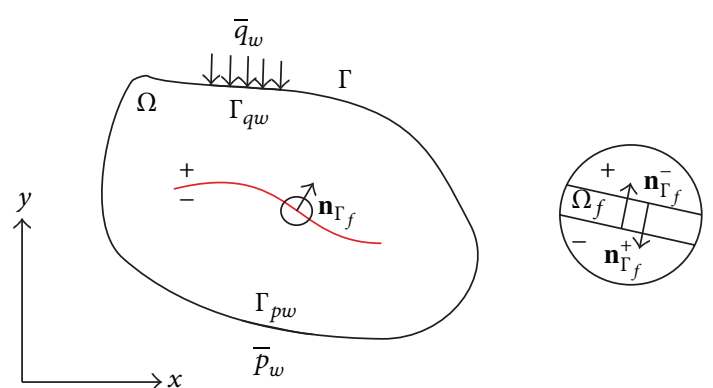

FIGURE 1: The domain and boundary for fractured porous media.

The initial and boundary conditions are as follows:

$$
\begin{gathered}
p^{w}=p^{w, 0} \quad \forall \mathbf{x} \in \Omega, \\
p_{m}^{w}(\mathbf{x})=\overline{p_{m}^{w}} \quad \mathbf{x} \in \Gamma_{p_{w}}, \\
\left(-\frac{\mathbf{K}_{m}}{\mu_{w}} \nabla p_{m}^{w}\right) \cdot \mathbf{n}_{\Gamma}=\frac{q_{w}}{\rho_{w}} \quad \forall \mathbf{x} \in \Gamma_{q_{w}} \\
p_{m}^{w}=p_{f}^{w} \quad \forall \mathbf{x} \in \Gamma_{f}, \\
\left(-\frac{\mathbf{K}_{m}}{\mu_{w}} \nabla p_{m}^{w}\right) \cdot \mathbf{n}_{\Gamma_{f}}=-\left(-\frac{K_{f}}{\mu_{w}} \nabla p_{f}^{w}\right) \cdot \mathbf{n}_{\Gamma_{f}}=\frac{q_{w}}{\rho_{w}},
\end{gathered}
$$

where $\mathbf{n}_{\Gamma}$ is the unit normal vector of the boundary, $\rho_{w}$ is fluid density, $\mathrm{kg} / \mathrm{m}^{3}$, and $q_{w}$ is the flow rate on the boundary, $\mathrm{kg} /\left(\mathrm{m}^{2} \cdot \mathrm{s}\right)$.

The weak forms of equations for flow in matrix and fracture are derived by weighted residual method

$$
\begin{aligned}
\int_{\Omega} \mathbf{w}_{p}^{T} & \left(\frac{\alpha_{m}-\phi_{m}}{K_{s}}+\frac{\phi_{m}}{K_{w}}\right) \frac{\partial p_{m}^{w}}{\partial t} d \Omega+\int_{\Omega}\left(\nabla \mathbf{w}_{p}\right)^{T} \frac{\mathbf{K}_{m}}{\mu_{w}} \nabla p_{m}^{w} d \Omega \\
& +\int_{\Omega} \mathbf{w}_{p}^{T} Q_{w s t c} B_{w} d \Omega+\int_{\Gamma_{f}^{+}} \mathbf{w}_{p}^{T} \frac{q_{w}^{+}}{\rho_{w}} d \Gamma+\int_{\Gamma_{f}^{-}} \mathbf{w}_{p}^{T} \frac{q_{w}^{-}}{\rho_{w}} d \Gamma \\
& +\int_{\Gamma_{q_{w}}} \mathbf{w}_{p}^{T} \frac{q_{w}}{\rho_{w}} d \Gamma=0,
\end{aligned}
$$

$$
\begin{aligned}
\int_{\Omega_{f}} \mathbf{w}_{p}^{T} \frac{1}{K_{w}} \frac{\partial p_{f}^{w}}{\partial t} d \Omega+\int_{\Omega_{f}}\left(\nabla \mathbf{w}_{p}\right)^{T} \frac{K_{f}}{\mu_{w}} \nabla p_{f}^{w} d \Omega-\int_{\Gamma_{f}^{+}} \mathbf{w}_{p}^{T} \frac{q_{w}^{+}}{\rho_{w}} d \Gamma \\
\quad-\int_{\Gamma_{f}^{-}} \mathbf{w}_{p}^{T} \frac{q_{w}^{-}}{\rho_{w}} d \Gamma=0,
\end{aligned}
$$

in which $\Omega_{f}$ is the fracture domain. The integrals over $\Omega_{f}$ are performed in the local Cartesian coordinate system $\left(x^{\prime}, y^{\prime}\right)$, whose $x^{\prime}$ axes and $y^{\prime}$ axes are consonant with tangential direction and normal direction, respectively.

With regard to the flow inside the fracture, both fluid pressure and its interpolating function are assumed to be uniform along fracture width [16], as shown in Figure 2. 


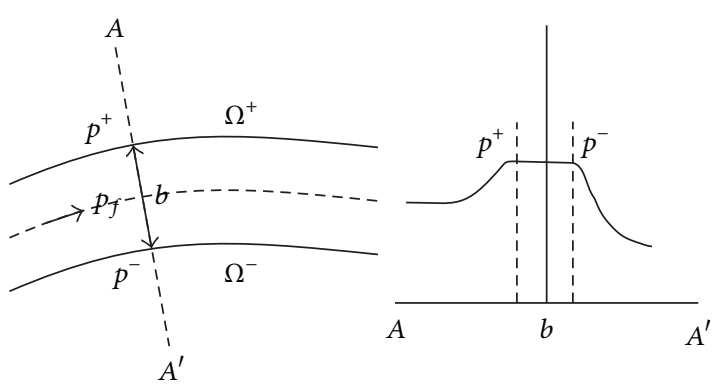

FIGURE 2: Fluid flow and pore fluid pressure around the fracture.

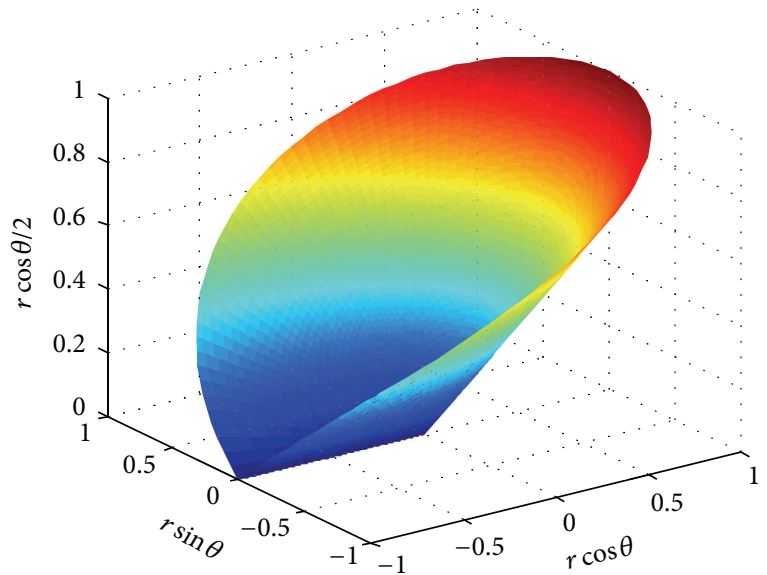

(a) The first branch function

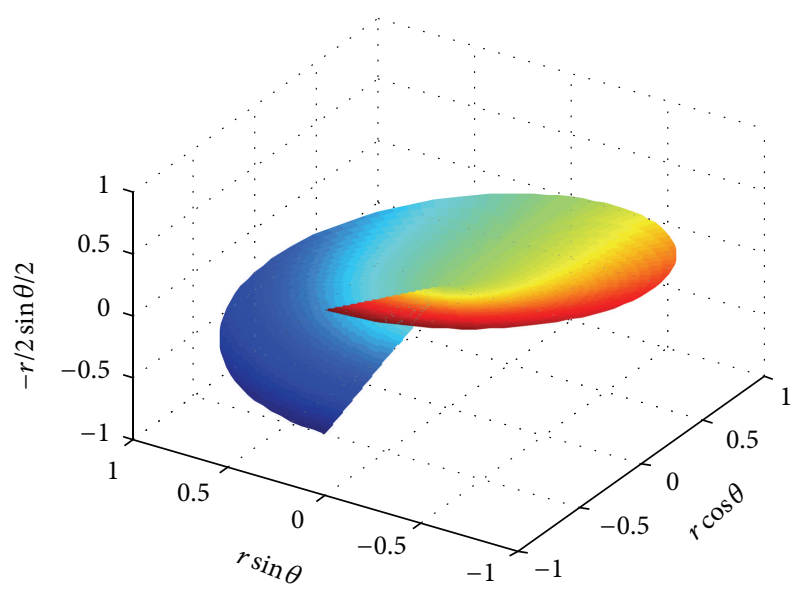

(b) Its derivative for pore pressure

FIgURE 3: The first branch function and its derivative for pore pressure.

Thus, the first integral of (6) can be rewritten as [16]

$$
\begin{aligned}
\int_{\Omega_{f}} \mathbf{w}_{p}^{T} \frac{1}{K_{w}} \frac{\partial p_{f}^{w}}{\partial t} d \Omega & =\int_{\Gamma_{f}} \int_{-b / 2}^{b / 2} \mathbf{w}_{p}^{T} \frac{1}{K_{w}} \frac{\partial p_{f}^{w}}{\partial t} d y^{\prime} d \Gamma \\
& =\int_{\Gamma_{f}} \mathbf{w}_{p}^{T} \frac{b}{K_{w}} \frac{\partial p_{f}^{w}}{\partial t} d \Gamma .
\end{aligned}
$$

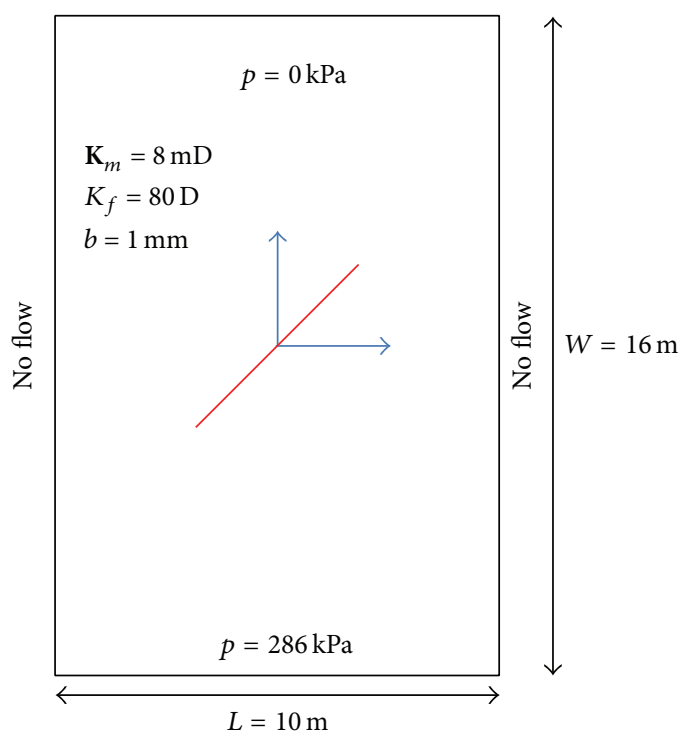

FIgURE 4: 2D fractured domain with applied pressure at top and bottom boundary.

Because of the supposed uniform distribution of fluid pressure on the $y^{\prime}$ axes, the derivative does not vary with $y^{\prime}$; thus the second integral is expressed as [16]

$$
\begin{aligned}
\int_{\Omega_{f}}\left(\nabla \mathbf{w}_{p}\right)^{T} \frac{K_{f}}{\mu_{w}} \nabla p_{f}^{w} d \Omega & =\int_{\Gamma_{f}} \int_{-b / 2}^{b / 2}\left(\nabla \mathbf{w}_{p}\right)^{T} \frac{K_{f}}{\mu_{w}} \nabla p_{f}^{w} d y^{\prime} d \Gamma \\
& =\int_{\Gamma_{f}} \frac{b K_{f}}{\mu_{w}} \frac{\partial w_{p}^{T}}{\partial x^{\prime}} \frac{\partial p_{f}^{w}}{\partial x^{\prime}} d \Gamma .
\end{aligned}
$$

Substituting the constituents of (6) and rearranging it, the weak form of continuity equation for flow inside fracture is gained. Adding it to the weak form for flow in matrix region, the fluid exchange term can be eliminated as (9) shows. Thus it is not necessary to express fluid exchange explicitly:

$$
\begin{aligned}
\int_{\Omega} \mathbf{w}_{p}^{T} & \left(\frac{\alpha_{m}-\phi_{m}}{K_{s}}+\frac{\phi_{m}}{K_{w}}\right) \frac{\partial p_{m}^{w}}{\partial t} d \Omega \\
& +\int_{\Omega}\left(\nabla \mathbf{w}_{p}\right)^{T}\left(\frac{\mathbf{K}_{m}}{\mu_{w}} \nabla p_{m}^{w}\right) d \Omega+\int_{\Gamma_{q_{w}}} \mathbf{w}_{p}^{T} \frac{q_{w}}{\rho_{w}} d \Gamma \\
& +\int_{\Gamma_{f}} \mathbf{w}_{p}^{T} \frac{b}{K_{w}} \frac{\partial p_{f}^{w}}{\partial t} d \Gamma+\int_{\Gamma_{f}} \frac{\partial \mathbf{w}_{p}^{T}}{\partial x^{\prime}} \frac{b K_{f} K_{r w}}{\mu_{w}} \frac{\partial p_{f}^{w}}{\partial x^{\prime}} d \Gamma=0 .
\end{aligned}
$$

\section{Pressure Enrichment with XFEM}

For permeable natural fracture, the pressure field is continuous at the fracture surface, while the derivative of pressure (i.e., flow rate) is discontinuous. The enrichment of the discontinuity in derivative is constructed as follows [21]. 


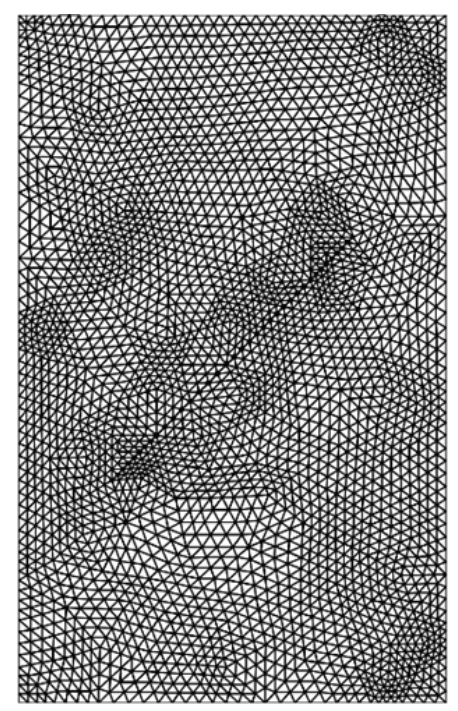

(a) DFM

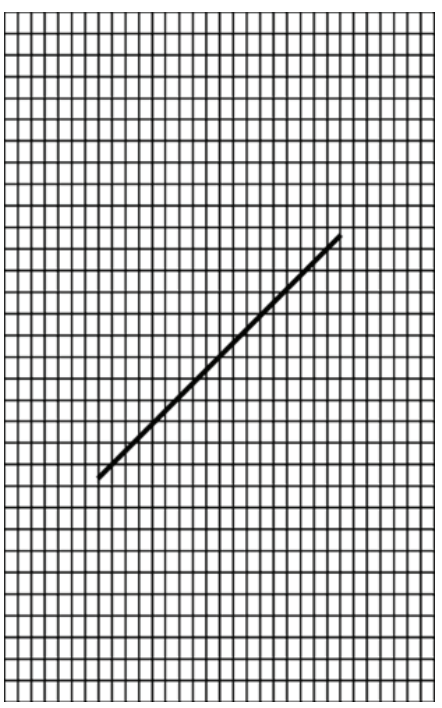

(b) FM-FEM

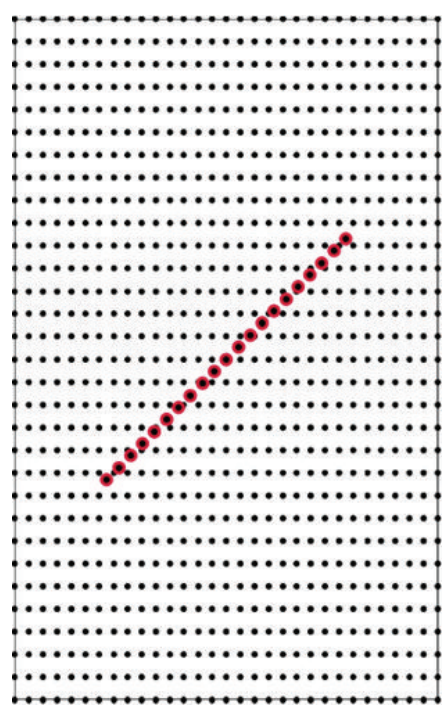

(c) FM-Mfree

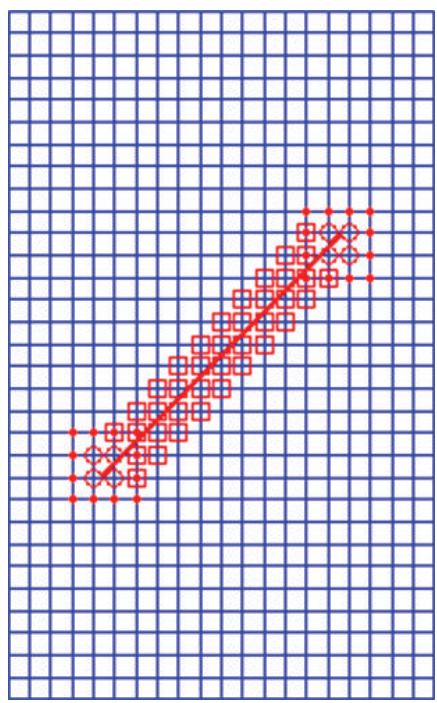

(d) XFEM

FIGURE 5: Domain discretization for different methods.

For a node with a bisected support, the absolute signed distance function is applied to realize the enrichment so as to meet the condition of continuous pressure and discontinuous derivative:

$$
F(\mathbf{x})=|f(\mathbf{x})| .
$$

For a support which is slit by the discontinuity, the branch function is applied to realize the enrichment:

$$
B^{l}(\mathbf{x})=\left[r \cos \frac{\theta}{2}, r^{2} \cos \frac{\theta}{2}, \sqrt{r} \cos \frac{\theta}{2}\right] .
$$

Its derivate with respect to $\theta$ is

$$
\frac{\partial B^{l}(\mathbf{x})}{\partial \theta}=\left[-\frac{r}{2} \sin \frac{\theta}{2},-\frac{r^{2}}{2} \sin \frac{\theta}{2},-\frac{\sqrt{r}}{2} \sin \frac{\theta}{2}\right],
$$

in which $\theta$ is the intersection angle between local coordinate system for fracture and global coordinate system.

The first branch function and its derivative for pore pressure are shown in Figure 3. On the surface of fracture (i.e., $\theta=\pi$ and $\theta=-\pi$ ), the interpolation function is continuous while its derivative is discontinuous. The other two interpolation functions share the same characteristics.

For the nodes cut by two intersecting discontinuities, the enrichment is realized through the product of two absolute signed distance functions related to the two discontinuities:

$$
J^{l}(\mathbf{x})=\left|f_{1}(\mathbf{x})\right|\left|f_{2}(\mathbf{x})\right|
$$

According to the fundamental XFEM, the local enrichment function and extra degrees of freedom are introduced 


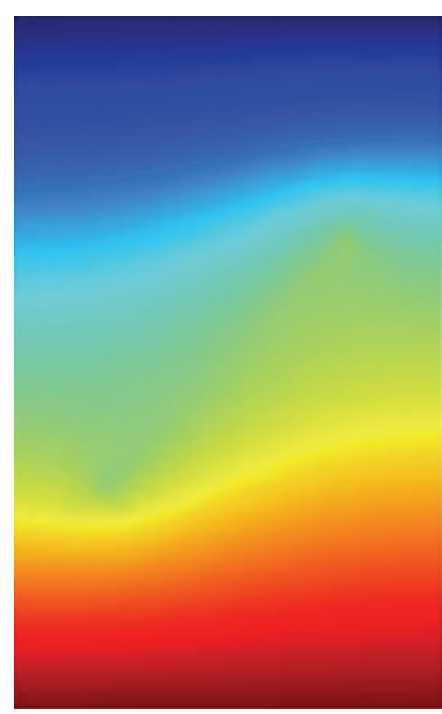

(a) DFM

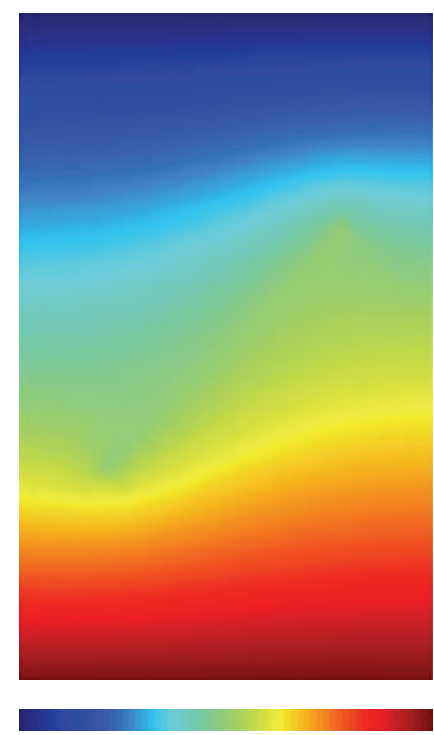

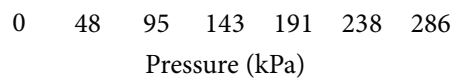

(c) FM-Mfree

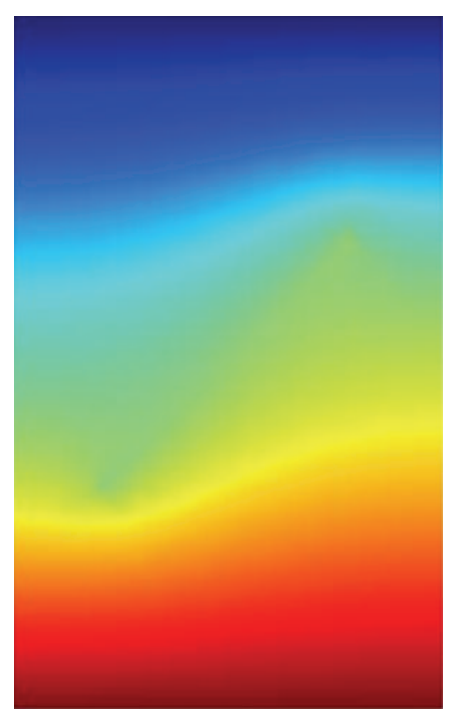

(b) FM-FEM

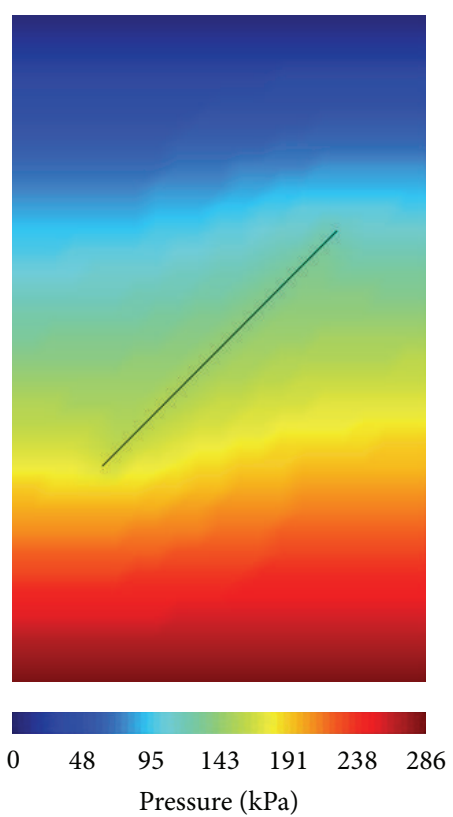

(d) XFEM

FIGURE 6: Pressure field obtained from different methods (independent fracture).

for interpolation of fluid pressure field. Then the pressure after enrichment can be expressed as

$$
p^{h}(\mathbf{x})=\sum_{i \in S} N_{i}(\mathbf{x}) p_{a i}+\underbrace{\sum_{j=1}^{N_{c}} \sum_{i \in S_{H j}} p_{b i, j} N_{i}(\mathbf{x})\left[\left|f_{j}(\mathbf{x})\right|-\left|f_{j}\left(\mathbf{x}_{i}\right)\right|\right]}_{\text {enrichment of bisected support for fracture } j}
$$$$
+\underbrace{\sum_{j=1}^{N_{t}} \sum_{i \in S_{C j}} N_{i}(\mathbf{x}) R^{j}(\mathbf{x})\left\{\sum_{l=1}^{3} p_{c i, j}^{l}\left[B_{j}^{l}(\mathbf{x})-B_{j}^{l}\left(\mathbf{x}_{i}\right)\right]\right\}}
$$
enrichment of slit support for fracture tip $j$

$$
+\underbrace{\sum_{j=1}^{N_{x}} \sum_{i \in S_{J j}} p_{d i, j} N_{i}(\mathbf{x})\left[J_{j}(\mathbf{x})-J_{j}\left(\mathbf{x}_{i}\right)\right]}_{\text {enrichment of intersecting support for cross point }},
$$

where $N_{i}$ is the node interpolation function, $R$ is the ramp function, $S$ is the set of all nodes, $S_{H j}$ is the set of nodes enriched by absolute signed distance function for fracture $j, S_{C j}$ is the set of nodes enriched by appropriate asymptotic function for fracture tip $j, S_{J j}$ is the set of nodes enriched by intersecting function for cross point $j, p_{a}$ is the freedom degree of regular nodes ( $a$-freedom degree), $p_{b}$ is the freedom 
degree of extra nodes in bisected support ( $b$-freedom degree), $p_{c}$ is the freedom degree of extra nodes in slit support ( $c$ freedom degree), and $p_{d}$ is the freedom degree of extra nodes in intersecting support ( $d$-freedom degree).

\section{Discretization Equation of XFEM}

Regarding trial function $\mathbf{w}_{p}$ as the variation of pore pressure interpolation function, the XFEM discretization equation of (9) in space is then gained considering the irrelevance of the values of $p_{a}, p_{b}, p_{c}$, and $p_{d}$ :

$$
\begin{aligned}
& \left(\left[\begin{array}{llll}
\mathbf{H}_{m, a a}^{w} & \mathbf{H}_{m, a b}^{w} & \mathbf{H}_{m, a c}^{w} & \mathbf{H}_{m, a d}^{w} \\
\mathbf{H}_{m, b a}^{w} & \mathbf{H}_{m, b b}^{w} & \mathbf{H}_{m, b c}^{w} & \mathbf{H}_{m, b d}^{w} \\
\mathbf{H}_{m, c a}^{w} & \mathbf{H}_{m, c b}^{w} & \mathbf{H}_{m, c c}^{w} & \mathbf{H}_{m, c d}^{w} \\
\mathbf{H}_{m, d a}^{w} & \mathbf{H}_{m, d b}^{w} & \mathbf{H}_{m, d c}^{w} & \mathbf{H}_{m, d d}^{w}
\end{array}\right]\right. \\
& \left.+\left[\begin{array}{llll}
\mathbf{H}_{f, a a}^{w} & \mathbf{H}_{f, a b}^{w} & \mathbf{H}_{f, a c}^{w} & \mathbf{H}_{f, a d}^{w} \\
\mathbf{H}_{f, b a}^{w} & \mathbf{H}_{f, b b}^{w} & \mathbf{H}_{f, b c}^{w} & \mathbf{H}_{f, b d}^{w} \\
\mathbf{H}_{f, c a}^{w} & \mathbf{H}_{f, c b}^{w} & \mathbf{H}_{f, c c}^{w} & \mathbf{H}_{f, c d}^{w} \\
\mathbf{H}_{f, d a}^{w} & \mathbf{H}_{f, d b}^{w} & \mathbf{H}_{f, d c}^{w} & \mathbf{H}_{f, d d}^{w}
\end{array}\right]\right\}\left\{\begin{array}{l}
\overline{p_{a}^{w}} \\
\overline{p_{a}^{w}} \\
\overline{p_{a}^{w}} \\
\overline{p_{a}^{w}}
\end{array}\right\} \\
& +\left(\left[\begin{array}{llll}
\mathbf{W}_{m, a a}^{w} & \mathbf{W}_{m, a b}^{w} & \mathbf{W}_{m, a c}^{w} & \mathbf{W}_{m, a d}^{w} \\
\mathbf{W}_{m, b a}^{w} & \mathbf{W}_{m, b b}^{w} & \mathbf{W}_{m, b c}^{w} & \mathbf{W}_{m, b d}^{w} \\
\mathbf{W}_{m, c a}^{w} & \mathbf{W}_{m, c b}^{w} & \mathbf{W}_{m, c c}^{w} & \mathbf{W}_{m, c d}^{w} \\
\mathbf{W}_{m, d a}^{w} & \mathbf{W}_{m, d b}^{w} & \mathbf{W}_{m, d c}^{w} & \mathbf{W}_{m, d d}^{w}
\end{array}\right]\right. \\
& \left.+\left[\begin{array}{cccc}
\mathbf{W}_{f, a a}^{w} & \mathbf{W}_{f, a b}^{w} & \mathbf{W}_{f, a c}^{w} & \mathbf{W}_{f, a d}^{w} \\
\mathbf{W}_{f, b a}^{w} & \mathbf{W}_{f, b b}^{w} & \mathbf{W}_{f, b c}^{w} & \mathbf{W}_{f, b d}^{w} \\
\mathbf{W}_{f, c a}^{w} & \mathbf{W}_{f, c b}^{w} & \mathbf{W}_{f, c c}^{w} & \mathbf{W}_{f, c d}^{w} \\
\mathbf{W}_{f, d a}^{w} & \mathbf{W}_{f, d b}^{w} & \mathbf{W}_{f, d c}^{w} & \mathbf{W}_{f, d d}^{w}
\end{array}\right]\right) \frac{d}{d t}\left\{\begin{array}{l}
\overline{p_{a}^{w}} \\
\overline{p_{a}^{w}} \\
\overline{p_{a}^{w}} \\
\overline{p_{a}^{w}}
\end{array}\right\} \\
& =\left\{\begin{array}{l}
\overline{q_{a}^{w}} \\
\overline{q_{a}^{w}} \\
\overline{q_{a}^{w}} \\
\overline{q_{a}^{w}}
\end{array}\right\},
\end{aligned}
$$

where flow matrix $\mathbf{H}$, compressibility matrix $\mathbf{W}$, flow rate vector $\mathbf{q}$ at the equivalent node, the unit tangent vector $\mathbf{I}_{f}^{t}$ of local coordinate system, and coordinate transform matrix $\mathbf{R}$ are, respectively, expressed as follows:

$$
\begin{array}{r}
\mathbf{H}_{m, \alpha \beta}^{w}=\int_{\Omega}\left(\nabla \mathbf{N}_{\alpha}^{p}\right)^{T} \frac{\mathbf{K}_{m}}{\mu_{w}} \nabla \mathbf{N}_{\beta}^{p} d \Omega \quad \alpha, \beta=a, b, c, d, \\
\mathbf{H}_{f, \alpha \beta}^{\pi}=\int_{\Gamma_{f}}\left(\nabla \mathbf{N}_{\alpha}^{p}\right)^{T} \mathbf{R}^{T} \mathbf{I}_{f}^{t} \frac{b K_{f}}{\mu_{w}}\left(\mathbf{I}_{f}^{t}\right)^{T} \mathbf{R} \nabla \mathbf{N}_{\beta}^{p} d \Gamma \\
\alpha, \beta=a, b, c, d,
\end{array}
$$

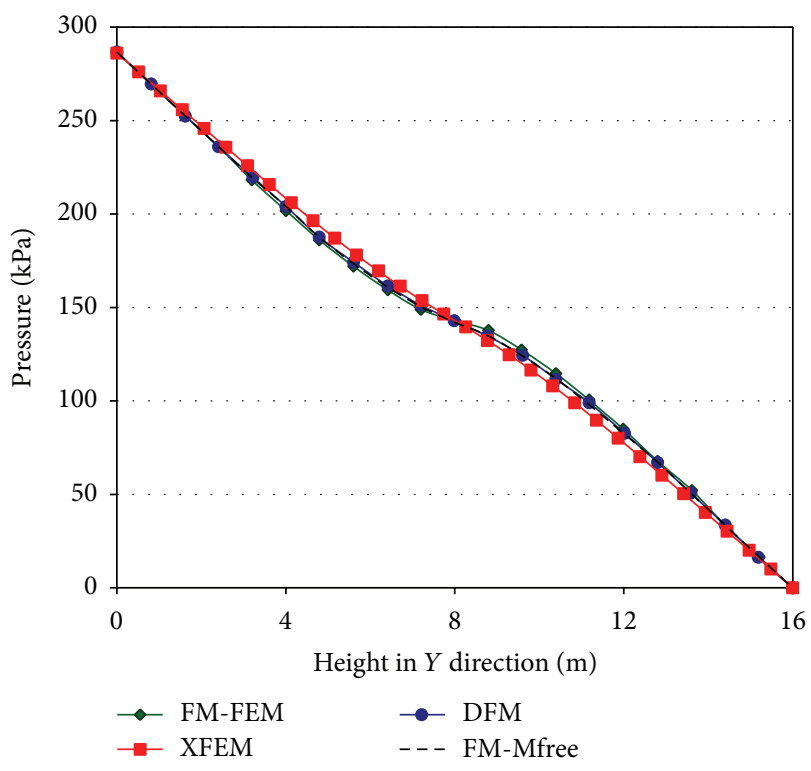

FiguRE 7: Pressure profile for a vertical section taken from the center of the domain.

$$
\begin{gathered}
\mathbf{W}_{m, \alpha \beta}^{w}=\int_{\Omega}\left(\mathbf{N}_{\alpha}^{p}\right)^{T}\left(\frac{\alpha_{m}-\phi_{m}}{K_{s}}+\frac{\phi_{m}}{K_{w}}\right) \mathbf{N}_{\beta}^{p} d \Omega \\
\alpha, \beta=a, b, c, d, \\
\mathbf{W}_{f, \alpha \beta}^{w}=\int_{\Gamma_{f}}\left(\mathbf{N}_{\alpha}^{p}\right)^{T} \frac{b}{K_{w}} \mathbf{N}_{\beta}^{p} d \Gamma \quad \alpha, \beta=a, b, c, d, \\
\overline{q_{\alpha}^{w}}=\int_{\Gamma_{q_{w}}}\left(\mathbf{N}_{\alpha}^{p}\right)^{T} \frac{q_{w}}{\rho_{w}} d \Gamma \quad \alpha=a, b, c, d, \\
\mathbf{I}_{f}^{t}=\left[\begin{array}{ll}
1 & 0
\end{array}\right]^{T}, \\
\mathbf{R}=\left[\begin{array}{cc}
\cos \theta & \sin \theta \\
-\sin \theta & \cos \theta
\end{array}\right] .
\end{gathered}
$$

Equation (15) can be rewritten as

$$
\mathbf{A X}+\mathbf{B} \frac{d \mathbf{X}}{d t}=\mathbf{C},
$$

in which $\mathbf{A}$ is the sum of matrix $\mathbf{H}_{m}$ of flow in matrix region and matrix $\mathbf{H}_{f}$ of flow in fracture, $\mathbf{B}$ is the sum of matrix $\mathbf{W}_{m}$ of rock compressibility and matrix $\mathbf{W}_{f}$ of fracture compressibility, $\mathbf{X}$ is the vector of pore pressure field, and $\mathbf{C}$ is the vector of flow rate at the equivalent node.

In the time domain, (17) can be discretized by the use of Newmark method as follows [22]:

$$
(\mathbf{B}+\Delta t \vartheta \mathbf{A}) \mathbf{X}_{n+1}=\left.\Delta t \mathbf{C}\right|_{n+\vartheta}+[\mathbf{B}-(1-\vartheta) \Delta t \mathbf{A}] \mathbf{X}_{n},
$$

in which parameter $\vartheta$ satisfies $0 \leq \vartheta \leq 1$. Only when $\vartheta \geq 1 / 2$, the solution is unconditionally stable, when $\vartheta=1 / 2$, it is the central difference in the time domain, and when $\vartheta=1$, it is fully implicit. 


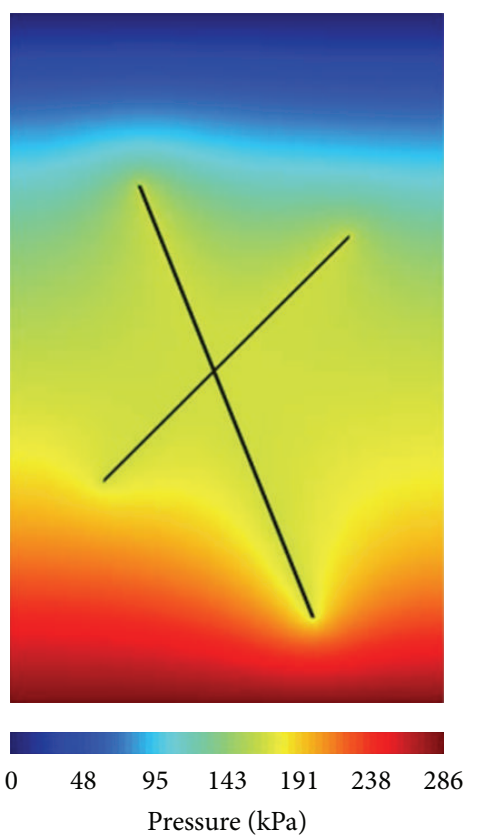

(a) DFM

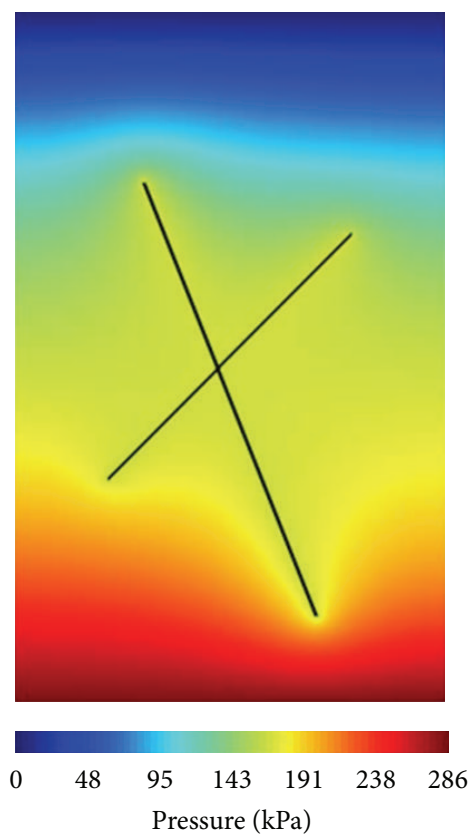

(b) FM-FEM

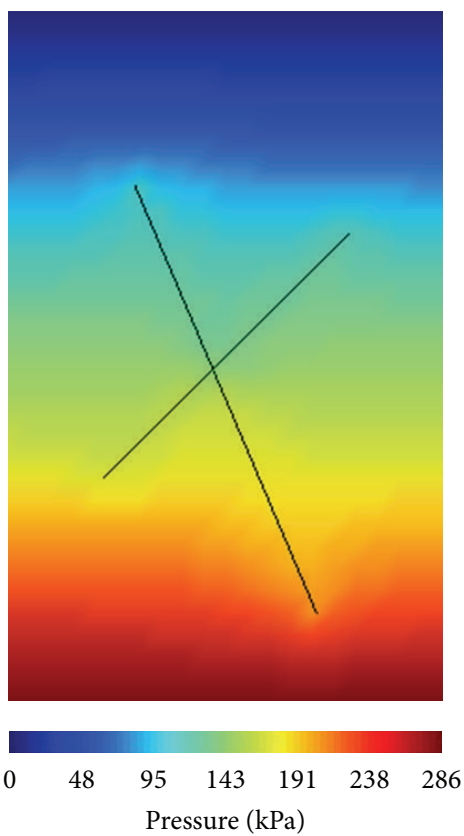

(c) XFEM

Figure 8: Pressure field obtained from different methods (intersecting fractures).

\section{Numerical Examples}

5.1. Steady State Flow. For a two-dimensional fractured domain with an independent fracture shown in Figure 4, Lamb [17] compared the calculated results of discrete fracture model (DFM), fracture mapping with mesh free (FM-Mfree), and fracture mapping with finite element method (FM-FEM) and verified the correctness of fracture mapping method. The domain discretization, pressure field, and pressure profile for a vertical section taken from the center of the domain for each method are, respectively, demonstrated in Figures 5, 6, and 7.

It is observed in Figure 5 that the mesh edges need to be set on the fracture and nodes need to be set on the fracture tip for DFM model, and the mesh node needs to be set on the fracture for FM-Mfree model. However, the FM-FEM and XFEM models can simulate the fluid flow in fractured porous medium with nonmatching grid. The permeability of elements with fracture is processed equivalently for FMFEM model, while the permeability of fracture is calculated only with cubic law or others for XFEM model. The red square represents the enriched node with a bisected support, hollow red circle represents the branch function enriched node with a silt support, and solid red circle represents the improved branch-function-enriched node for XFEM, as shown in Figure 5(d) [23].

As can be seen from Figures 6 and 7, the calculated pressure distribution and pressure profile are basically the same for 4 methods with different cell number and node number. From this, the XFEM approach proposed in this paper is verified.

Because of the introduction of enrichment functions with discontinuous derivate and extra degrees of freedom for node, it is not necessary to consider the location of fracture

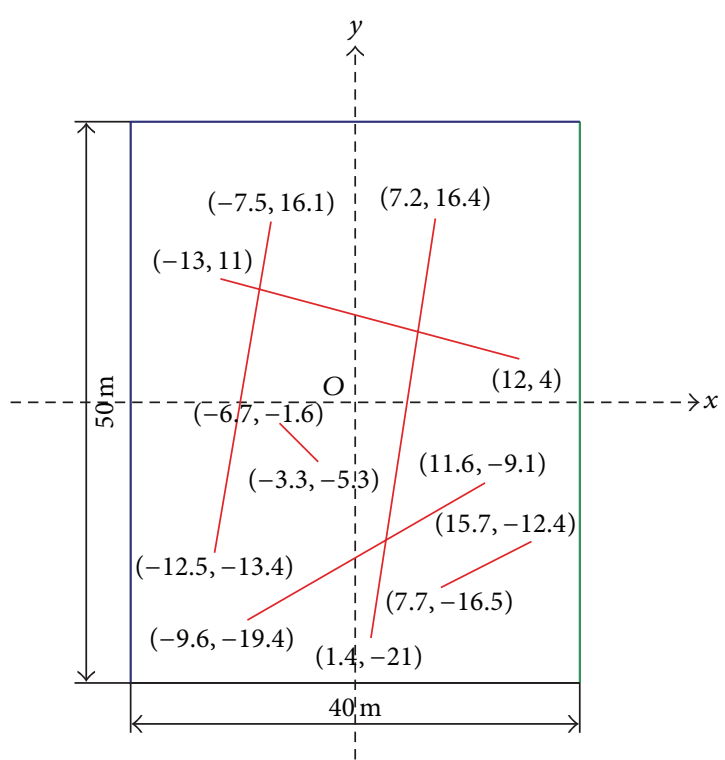

FIgURE 9: Domain and boundary conditions for single phase fluid flow.

in the process of domain discretization, which makes it more convenient when treating complex network fractures. In particular, when fracture propagation is concerned such as that during hydraulic fracturing process, there is no need to remesh and transform nodal pressure filed, with obvious merit.

Adding one more fracture in the domain shown in Figure 4, it becomes intersecting fracture with the rest of parameters and boundary conditions unchanged. Lamb [17] 


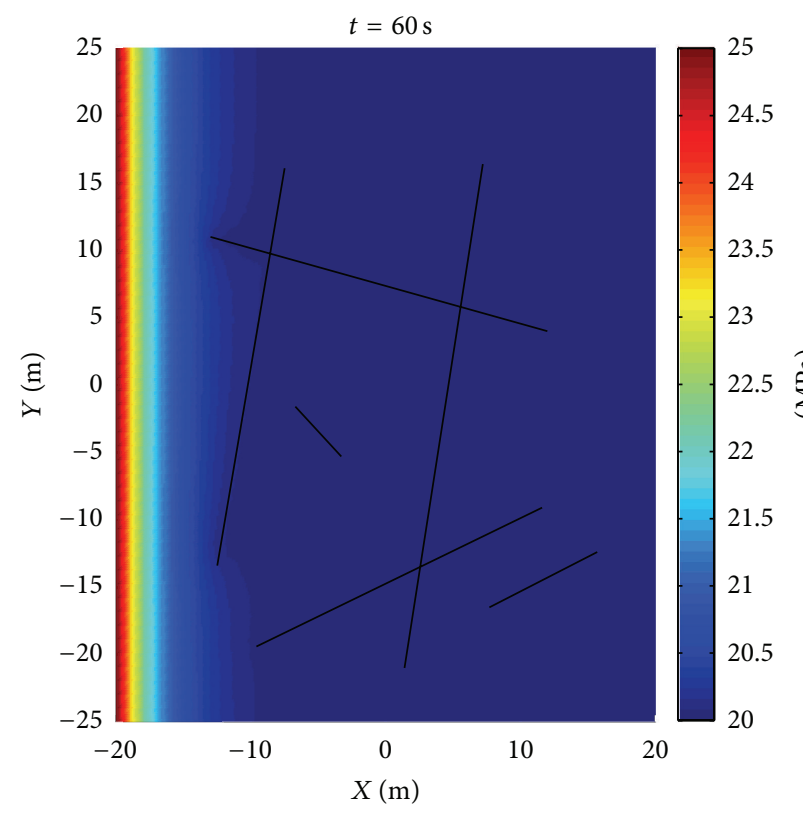

(a) $t=60 \mathrm{~s}$

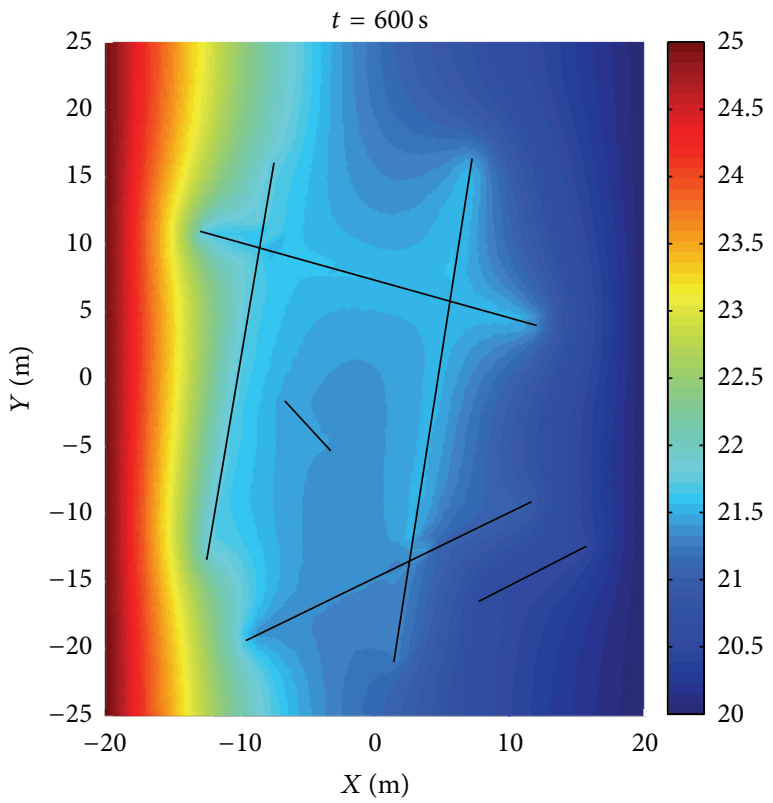

(c) $t=600 \mathrm{~s}$

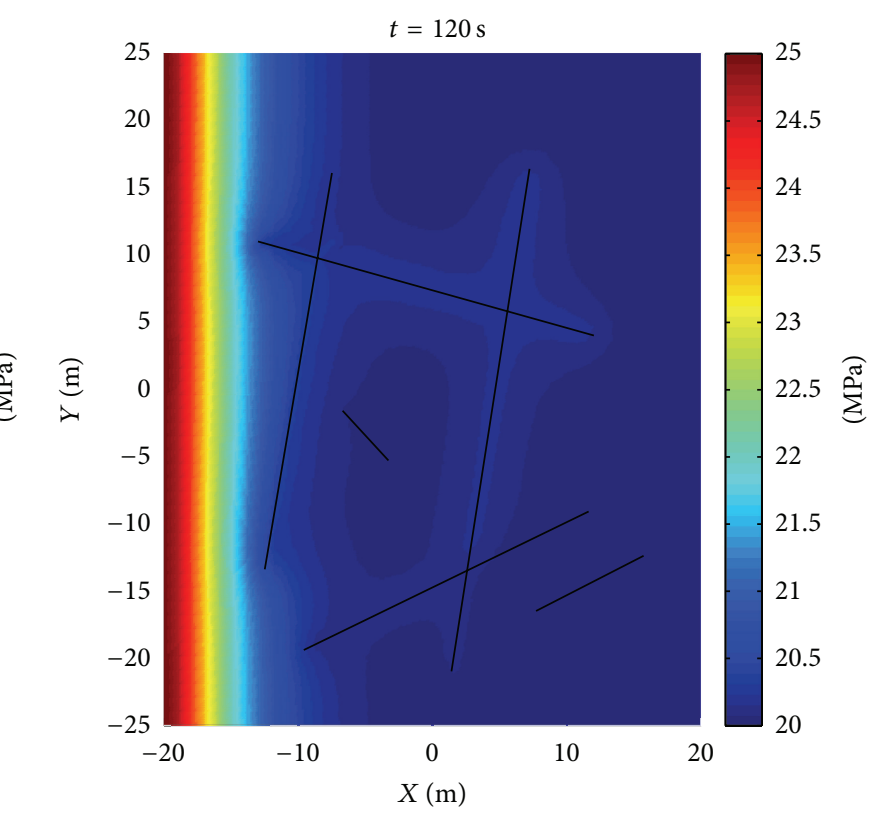

(b) $t=120 \mathrm{~s}$

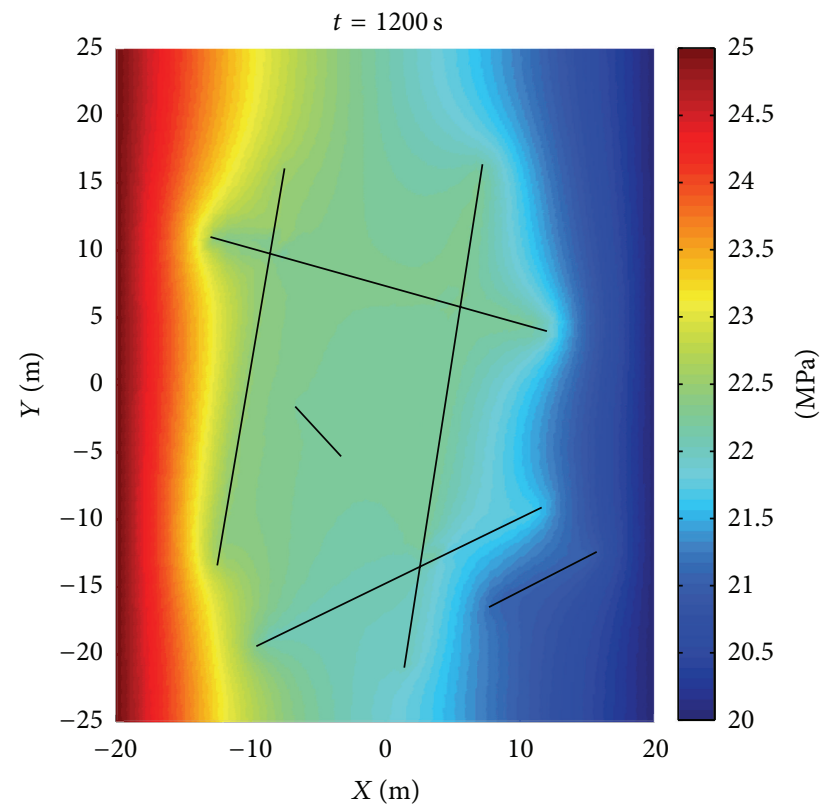

(d) $t=1200 \mathrm{~s}$

FIgure 10: Pore fluid pressure distribution at different moments.

computed the pressure distributions in the existence of intersecting fractures by use of DFM and FM-FEM, respectively. Comparing them in Figure 8 with the result using XFEM, it is recognized that the result of XFEM has a good consistency with that of DFM and FM-FEM, which confirms that the fractured-porous-media flow model proposed in this paper can describe not only the flow process in both fracture and matrix but also process independent and intersecting fractures. Thus, it builds a solid foundation for coupled HM simulation and analysis of fracture propagation during volume stimulation under the concept of XFEM.
5.2. Transient State Flow. Consider a 40 meters long and 50 meters wide horizontal reservoir with several 1.5-millimeterswide fractures as shown in Figure 9. The permeability and porosity of matrix rock is $100 \times 10^{-15} \mathrm{~m}^{2}$ and 0.18 , respectively. The initial pore fluid pressure is $20 \mathrm{MPa}$. Both the lower boundary and the upper boundary are impermeable. The left boundary has a constant pressure of $25 \mathrm{MPa}$, while the right boundary has a constant pressure of $20 \mathrm{MPa}$. The bulk modulus of water phase and solid rock grains is $2.0 \mathrm{GPa}$ and $134.6 \mathrm{GPa}$, respectively. The viscosity of water phase is $5 \mathrm{mPa} \cdot \mathrm{s}$. 
Applying $39 \times 49$ structured gird, the distributions of pore fluid pressure at different moments $(t=60 \mathrm{~s}, t=120 \mathrm{~s}$, $t=600 \mathrm{~s}$, and $t=1200 \mathrm{~s}$ ) are demonstrated in Figure 10 after calculating with XFEM. Before pressure spreading to fracture $(t<60 \mathrm{~s})$, the isobaric lines appear to be parallel, which is in the stage of linear displacement (Figure 10(a)). During pressure encountering fracture $(t>60 \mathrm{~s})$, the isobaric line bents toward tip direction near the fracture tip, and the fracture tip is presented as a point/sink source at the moment (Figures 10(b) 10(d)). After pressure sweeping over the whole fractured region, the pressure drop of whole fractured region is very small, and pressure loss occurs mainly in the matrix (Figures 10(c) and 10(d)).

\section{Conclusions and Discussions}

(1) Both absolute signed distance function and appropriate asymptotic function are continuous but discontinuous in derivatives, which enables them to be enrichment functions during processing pressure field across permeable fractures.

(2) Based on the explicit expression of real distribution of fractures, locally enrichment with XFEM was used to develop fluid flow in fractured porous medium. The model is able to elaborately simulate the flow in reservoir with arbitrary independent, kinking, branching, intersecting, and complex network fractures.

(3) Domain discretization is independent of fracture location with XFEM flow model. Moreover, remeshing is unnecessary when dealing with dynamic propagating fractures, which enables it to be used for simulating hydraulic fracturing with coupled HM model.

\section{Conflict of Interests}

The authors declare that there is no conflict of interests regarding the publication of this paper.

\section{Acknowledgment}

The authors are grateful for the research support of the National Natural Science Foundation of China (NSFC, no. 51404207).

\section{References}

[1] B. Berkowitz, J. Bear, and C. Braester, "Continuum models for contaminant transport in fractured porous formations," Water Resources Research, vol. 24, no. 8, pp. 1225-1236, 1988.

[2] G. I. Barenblatt, I. P. Zheltov, and I. N. Kochina, "Basic concepts in the theory of seepage of homogeneous liquids in fissured rocks [strata]," Journal of Applied Mathematics and Mechanics, vol. 24, no. 5, pp. 1286-1303, 1960.

[3] M. Karimi-Fard, L. J. Durlofsky, and K. Aziz, "An efficient discrete-fracture model applicable for general-purpose reservoir simulators," SPE Journal, vol. 9, no. 2, pp. 227-236, 2004.
[4] J. Lee, S.-U. Choi, and W. Cho, "A comparative study of dualporosity model and discrete fracture network model," KSCE Journal of Civil Engineering, vol. 3, no. 2, pp. 171-180, 1999.

[5] S. H. Lee, M. F. Lough, and C. L. Jensen, "Hierarchical modeling of flow in naturally fractured formations with multiple length scales," Water Resources Research, vol. 37, no. 3, pp. 443-455, 2001.

[6] B. G. Chen, E. X. Song, and X. H. Cheng, "A numerical method for discrete fracture network model for flow and heat transfer in two-dimensional fractured rocks," Chinese Journal of Rock Mechanics and Engineering, vol. 33, no. 1, pp. 43-51, 2014.

[7] J. E. Warren and P. J. Root, "The behavior of naturally fractured reservoirs," Old SPE Journal, vol. 3, no. 3, pp. 245-255, 1963.

[8] K. Pruess and T. N. Narasimhan, "A practical method for modeling fluid and heat flow in fractured porous media," Society of Petroleum Engineers journal, vol. 25, no. 1, pp. 14-26, 1985.

[9] O. Kolditz, "Modelling flow and heat transfer in fractured rocks: conceptual model of a 3-D deterministic fracture network," Geothermics, vol. 24, no. 3, pp. 451-470, 1995.

[10] J. Yao, Z. S. Wang, Y. Zhang, and Z. Q. Huang, "Numerical simulation method of discrete fracture network for naturally fractured reservoirs," Acta Petrolei Sinica, vol. 31, no. 2, pp. $284-$ 288, 2010 (Chinese).

[11] B. L. Karihaloo and Q. Z. Xiao, "Modelling of stationary and growing cracks in FE framework without remeshing: a state-ofthe-art review," Computers \& Structures, vol. 81, no. 3, pp. 119$129,2003$.

[12] N. Moës, J. Dolbow, and T. Belytschko, "A finite element method for crack growth without remeshing," International Journal for Numerical Methods in Engineering, vol. 46, no. 1, pp. 131-150, 1999.

[13] L. X. Li and T. J. Wang, "The extended finite element method and its applications: a review," Advances in Mechanics, vol. 35, no. 1, pp. 5-20, 2005.

[14] T. T. Yu, The Extended Finite Element Method: Theory, Application and Program, Science Press, Beijing, China, 2014, (Chinese).

[15] A. R. Khoei, S. Moallemi, and E. Haghighat, "Thermo-hydromechanical modeling of impermeable discontinuity in saturated porous media with X-FEM technique," Engineering Fracture Mechanics, vol. 96, pp. 701-723, 2012.

[16] T. Mohammadnejad and A. R. Khoei, "An extended finite element method for hydraulic fracture propagation in deformable porous media with the cohesive crack model," Finite Elements in Analysis and Design, vol. 73, pp. 77-95, 2013.

[17] A. R. Lamb, Coupled Deformation, Fluid Flow and Fracture Propagation in Porous Media, Imperial College, London, UK, 2011.

[18] A. R. Lamb, G. J. Gorman, and D. Elsworth, "A fracture mapping and extended finite element scheme for coupled deformation and fluid flow in fractured porous media," International Journal for Numerical and Analytical Methods in Geomechanics, vol. 37, no. 17, pp. 2916-2936, 2013.

[19] N. Watanabe, W. Wang, J. Taron, U. J. Görke, and O. Kolditz, "Lower-dimensional interface elements with local enrichment: application to coupled hydro-mechanical problems in discretely fractured porous media," International Journal for Numerical Methods in Engineering, vol. 90, no. 8, pp. 1010-1034, 2012.

[20] P. A. Witherspoon, J. S. Y. Wang, K. Iwai, and J. E. Gale, "Validity of cubic law for fluid flow in a deformable rock fracture," Water Resources Research, vol. 16, no. 6, pp. 1016-1024, 1980. 
[21] T. Belytschko, N. Moes, S. Usui, and C. Parimi, "Arbitrary discontinuities in finite elements," International Journal for Numerical Methods in Engineering, vol. 50, no. 4, pp. 993-1013, 2001.

[22] R. W. Lewis and B. A. Schrefler, The Finite Element Mothod in the Static and Dynamic Deformation and Consolidation of Porous Media, John Wiley \& Sons, 1998.

[23] T. T. Yu and Z. W. Gong, "Determination of enrichment type of node in extended finite element method," Rock and Soil Mechanics, vol. 34, no. 11, pp. 3284-3290, 2013 (Chinese). 


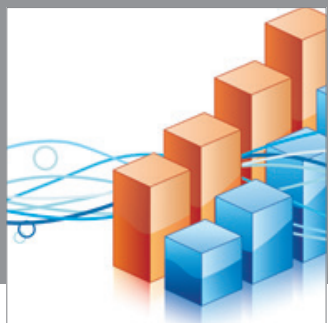

Advances in

Operations Research

mansans

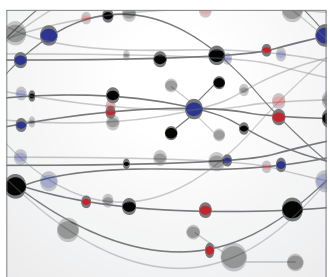

The Scientific World Journal
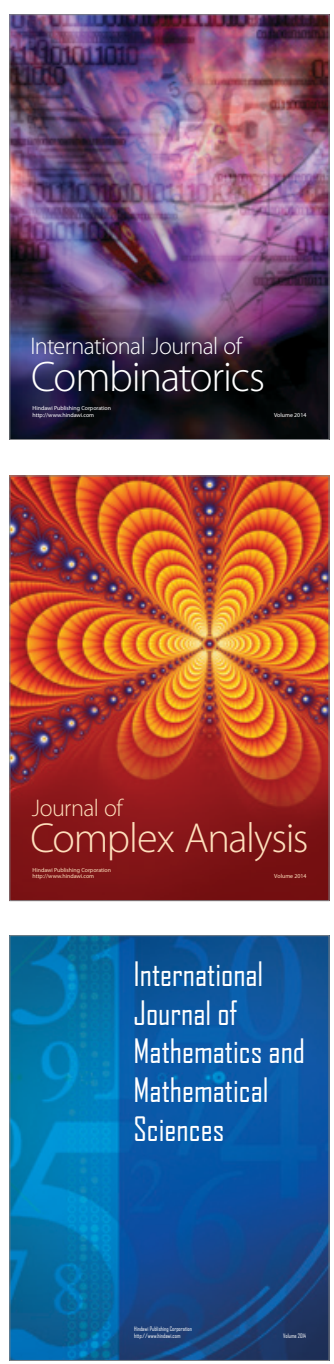
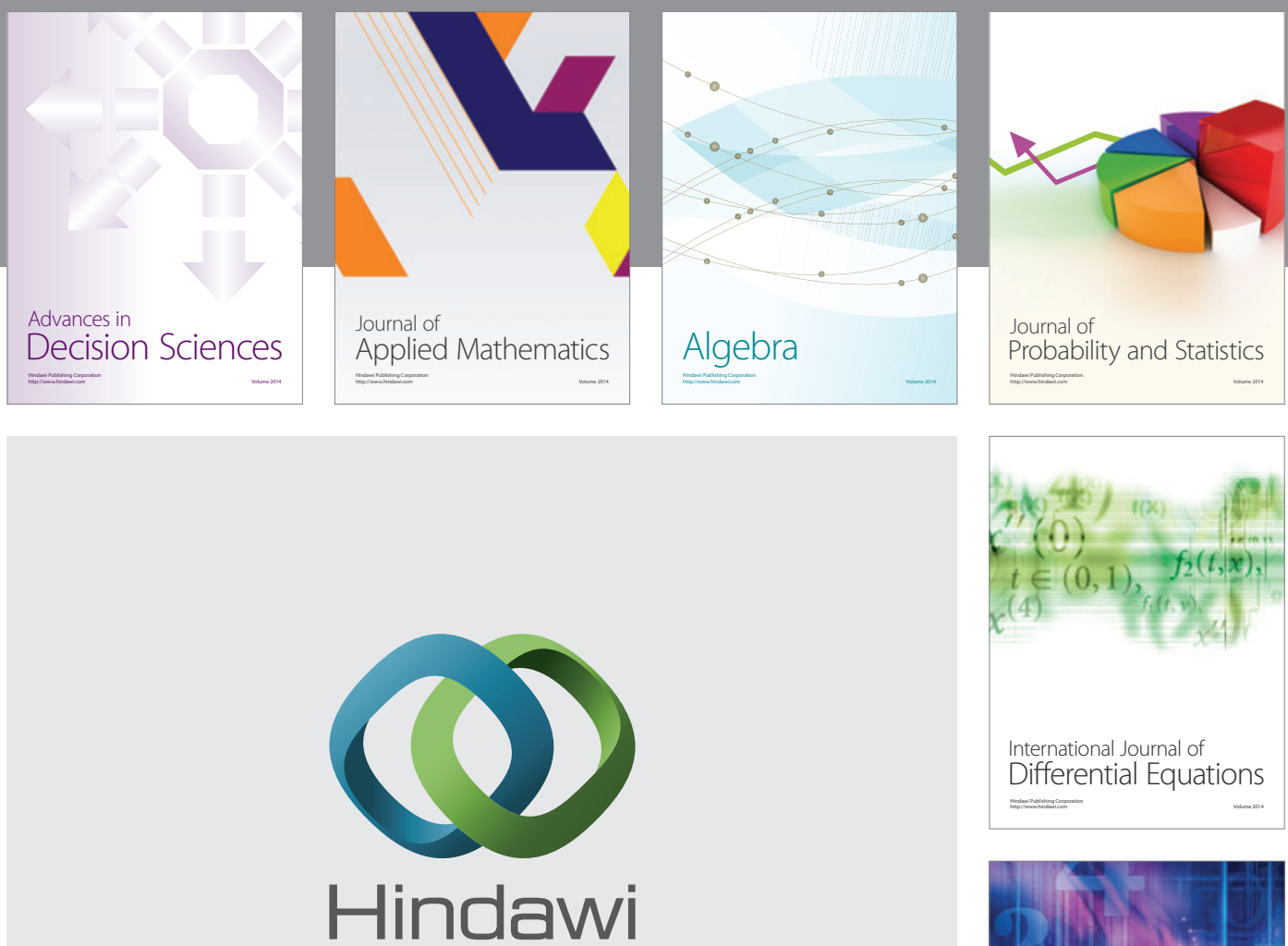

Submit your manuscripts at http://www.hindawi.com
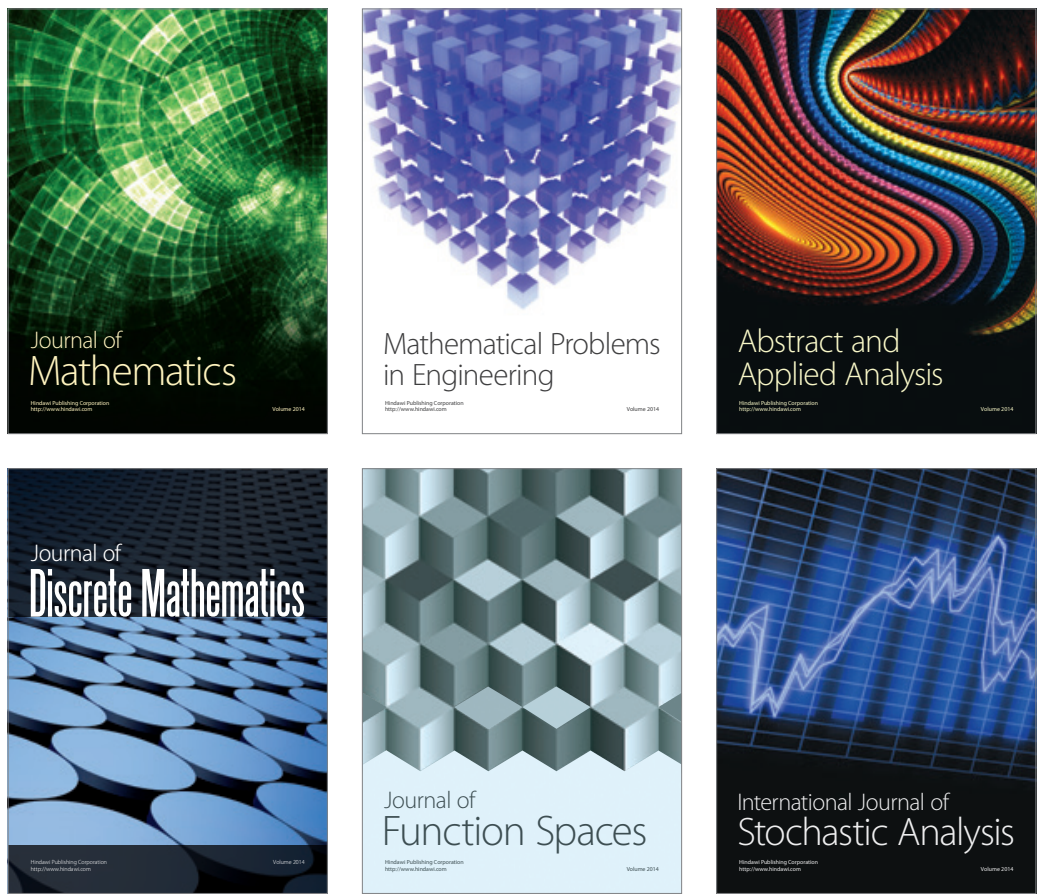

Journal of

Function Spaces

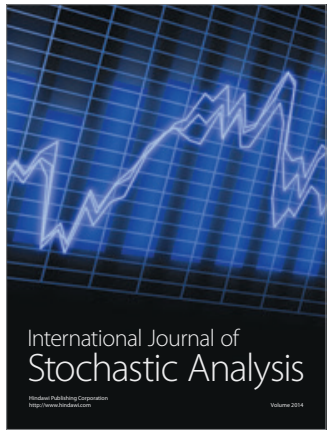

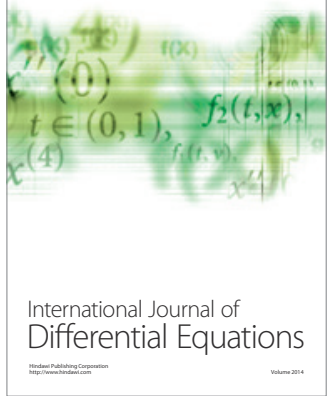
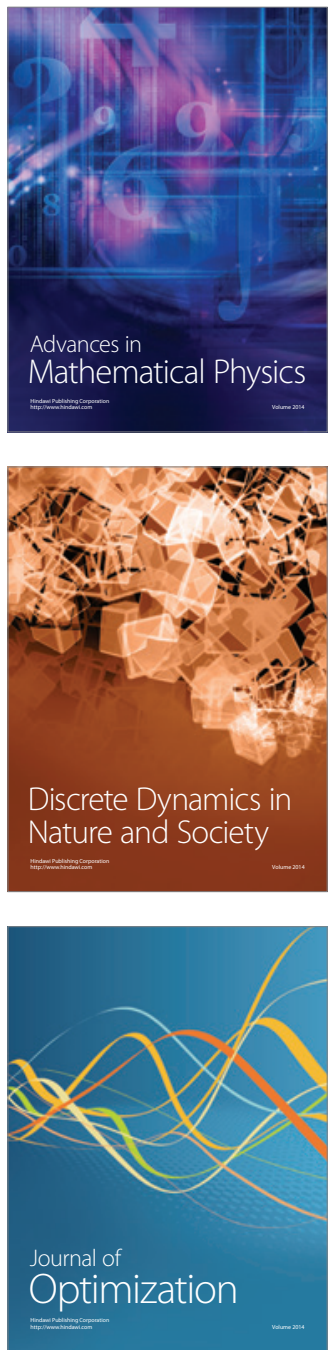